Indian J. Pure Appl. Math., 51(2): 705-715, June 2020

(c) Indian National Science Academy

DOI: $10.1007 / \mathrm{s} 13226-020-0425-5$

\title{
SOME RESULTS ON NOETHERIAN AND ARTINIAN BL-ALGEBRAS
}

\author{
Jamal Kazemiasl, Farhad Khaksar Haghani and Shahram Heidarian \\ Department of Mathematics, Shahrekord Branch, \\ Islamic Azad University, Shahrekord, Iran \\ e-mails: kazemiasl.j@gmail.com; haghani1351@yahoo.com; \\ heidarianshm@gmail.com \\ (Received 27 January 2018; after final revision 25 March 2018; \\ accepted 2 April 2019)
}

\begin{abstract}
In this paper, we obtain some relations between Noetherian and Artinian BL-algebras. Further, we derive some theorems and lemmas for composition series, Artinian BL-argebera, as well as the relations between Noetherian, Artinian and short exact sequences. We further study the Noetherian and Artinian over homomorphism BL-algebras and obtain some new results concerning to the essential deductive systems of BL-algebras.
\end{abstract}

Key words : Artinian (Noetherian) BL-algebra; composition series; maximal filter; short exact sequence.

2010 Mathematics Subject Classification : 03G25, 03G99, 06D99.

\section{INTRODUCTION}

BL-algebras are the algebraic structures for Hájek [3] basic logic in order to investigate many-valued logic by algebraic means. He provided an algebraic counterpart of a propositional logic, called Basic Logic, which embodies a fragment common to some of the most important many-valued logics, namely Lukasiewicz Logic, Gödel Logic and Product Logic [1]. This Basic Logic (BL for short) is proposed as the most general many-valued logic with truth values in interval $[0,1]$ and BL-algebras are the corresponding Lindenbaum Tarski algebras. Also, he provided an algebraic mean to study continuous t-norms (or triangular norms) in [0, 1], [3]. The language of propositional Hájek basic logic (1998) contains the binary connectives $o$ and $\Rightarrow$ and the constant $\overline{0}$. Axioms of BL are given as:

(A1) $(\varphi \Rightarrow \psi) \Rightarrow((\psi \Rightarrow w) \Rightarrow(\varphi \Rightarrow w))$. 
(A2) $(\varphi \circ \psi) \Rightarrow \varphi$.

(A3) $(\varphi \circ \psi) \Rightarrow(\psi \circ \varphi)$.

(A4) $(\varphi \circ(\varphi \Rightarrow \psi)) \Rightarrow(\psi o(\psi \Rightarrow \varphi))$.

(A5a) $(\varphi \Rightarrow(\psi \Rightarrow w)) \Rightarrow((\varphi \circ \psi) \Rightarrow w)$.

(A5b) $((\varphi \circ \psi) \Rightarrow w) \Rightarrow(\varphi \Rightarrow(\psi \Rightarrow w))$.

(A6) $((\varphi \Rightarrow \psi) \Rightarrow w) \Rightarrow((\psi \Rightarrow \varphi) \Rightarrow w) \Rightarrow w)$.

(A7) $\overline{0} \Rightarrow w$.

Hájek [3] introduced the concepts of filters and prime filters in BL-algebras. From logical point of view, filters correspond to sets of provable formula. E. Turunen studied some properties of filters theory, which plays important role in studying logical algebras. He showed how BL-algebras can be studied by deductive systems. Deductive systems correspond to subsets closed with respect to Modus Ponens and they are called filters, too. Motamed and Moghaderi [6], introduced the notions of Noetherian and Artinian on BL-algebras. They obtained some equivalent definitions of Noetherian and Artinian BL-algebras and proved the Anderson and Cohen Theorems of rings theory in BLalgebra. The same authors also introduced the short exact sequences in BL-algebras. In this paper, we obtain some new results following [6].

The structure of the paper is as follows. In Section 2, we recall some definitions and results about BL-algebras that we will use in the sequel. In Section 3, we define the notion of Noetherian and Artinian BL-algebras and we drive some results about the relations between Noetherion and Artinian, composition series, radical of a BL-algebra, finitely generated filters, short exact sequences and essential deductive systems in BL-algebras.

\section{PRELIMINARIES}

In this section, we recall and review some definitions and results relevant to Noetherian (Artinian), composition series, short exact sequence BL-algebra, which will be used throughout of the paper.

An algebra $(A, \wedge, \vee, \odot, \longrightarrow, 0,1)$ of the type $(2,2,2,2,0,0)$ is called a BL-algebra if for all $a, b, c \in A$ satisfy the following axioms:

(BL1) $(A, \wedge, \vee, 0,1)$ is a bounded lattice.

(BL2) $(A, \odot, 1)$ is a commutative monoid.

$($ BL3) $\odot$ and $\longrightarrow$ form an adjoint pair, i.e., $c \leq a \longrightarrow b$ if and only if $a \odot c \leq b$. 
(BL4) $a \wedge b=a \odot(a \longrightarrow b)$.

(BL5) $(a \longrightarrow b) \vee(b \longrightarrow a)=1$.

We will denote $\bar{x}=x \longrightarrow 0$ and $x^{--}=(\bar{x})^{-}$, for all $x \in A$.

Examples of BL-algebras [3] are t-algebras $([0,1], \wedge, \vee, \odot, \longrightarrow, 0,1)$ where $([0,1], \wedge, \vee, 0,1)$ is the usual lattice on $[0,1]$ and $\odot$, is a continuous t-norm, whereas $\rightarrow$, is the corresponding residuum.

Throughout of this paper by $A$, we denote the universe of a BL-algebra.

A BL-algebra is nontrivial if $0 \neq 1$. For any BL-algebra $A$, the reduct $L(A)=(A, \wedge, \vee, 0,1)$ is a bounded distributive lattice. We denote the set of natural numbers by $\mathbb{N}$ and define $a^{0}=1$ and $a^{n}=a^{n-1} \odot a$, for $n \in \mathbb{N} \backslash\{0\}$.

Hájek [3] defined a filter of a BL-algebra $A$ to be a nonempty subset $F$ of $A$ such that $(i)$ if $a, b \in F$ implies $a \odot b \in F(i i)$ if $a \in F, a \leq b$ then $b \in F$. E. Turunen [8] defined a deductive system of a BL-algebra $A$ to be a nonempty subset $D$ of $A$ such that $(i) 1 \in D$ and $(i i) x \in D$ and $x \longrightarrow y \in D$ imply $y \in D$. Note that a subset $F$ of a BL-algebra $A$ is a deductive system of $A$ if and only if $F$ is a filter of $A$ [8]. Let $F$ be a filter of a BL-algebra $A$, then $F$ is proper filter if $F \neq A$.

A proper filter $P$ of $A$ is called a prime filter of $A$ if for all $x, y \in A, x \vee y \in P$ implies $x \in P$ or $y \in P$. A proper filter $P$ of $A$ is prime if and only if $P$ can not be expressed as an intersection of two filters properly containing $P$ or equivalently, for all $x, y \in A$, either $x \longrightarrow y \in P$ or $y \longrightarrow x \in P$ [8].

If $F, G$ and $P$ are filters of $A$, then $P$ is a prime filter of $A$ if and only if $F \cap G \subseteq P$ implies $F \subseteq P$ or $G \subseteq P$.

In [8], it can be seen that a proper filter $M$ of $A$ is a maximal filter of $A$ if and only if for all $x \notin M$, there exists $n \in \mathbb{N}$ such that $\left(x^{n}\right)^{-} \in M$. Every maximal filter of $A$ is a prime filter of $A$ [8].

The set of all filters, all prime filters of a BL-algebra $A$ and all maximal filters of a BL-algebra $A$ are denote by $\digamma(A), \operatorname{Spec}(A)$ and $\operatorname{Max}(A)$, respectively. The filter of $A$ generated by $X$ is denoted by $\langle X\rangle$, where $X \subseteq A$, in which $\langle\emptyset\rangle=\{1\}$ and $\langle X\rangle=\left\{a \in A: x_{1} \odot x_{2} \odot, \ldots, \odot x_{n} \leq a\right.$, for some $\mathrm{n} \in \mathbb{N}$ and $\left.x_{1}, x_{2}, \ldots, x_{n} \in X\right\}[5,8]$.

$F \in \digamma(A)$ is called a finitely generated filter, if $F=\left\langle x_{1}, \ldots, x_{n}\right\rangle$, for some $x_{1}, \ldots, x_{n} \in A$ and $n \in \mathbb{N}$. For $F \in F(A)$ and $x \in A \backslash F$, define $F\langle x\rangle=\langle F \cup\{x\}\rangle$ or equally $F\langle x\rangle=\{a \in A: a \geq$ $f \odot x^{n}$, for some $f \in F$, and $n \geq 1$. $\}$

Remark 2.1 : [2]. Let $F$ and $G$ be two filters of $A$ such that $F \subseteq G$. It is evident that $\frac{G}{F}$ is a 
filter of $\frac{A}{F}$. Since $G$ is a filter, then it can be easily shown that $\frac{a}{F} \in \frac{G}{F}$ if and only if a $\in G$. Hence, $\digamma\left(\frac{A}{F}\right)=\left\{\frac{H}{F}: \mathrm{H} \in \digamma(A), F \subseteq H\right\}$.

Theorem 2.2 - [8]. Each maximal filter $F$ of a distributive lattice A is a prime filter.

Definition $2.3-$ [8]. Let $A$ and $B$ be two BL-algebras. A map $f: A \longrightarrow B$ defined on $A$, is called a BL-homomorphism if, for all $x, y \in A, f(x \longrightarrow y)=f(x) \longrightarrow f(y), f(x \odot y)=f(x) \odot$ $f(y)$ and $f\left(0_{A}\right)=0_{B}$. Also, define $\operatorname{ker}(f)=\{a \in A: f(a)=1\}$ and $\operatorname{Im}(f)=\{f(a): a \in A\}$.

Definition 2.4 - [9]. Let $F$ be a filter of BL-algebra $A$. By an $F$-chain we mean a sequence $\left\{F_{i} \mid i=0,1,2, \ldots, n\right\}$ of filters of $A$ such that

$$
\{1\}=F_{0} \subset F_{1} \subset F_{2} \subset \cdots \subset F_{n-1} \subset F_{n}=F,
$$

where $\subset$ is a strict inclusion, and $n$ indicates the length of this chain. An $F$-chain $\left\{F_{i}: i=\right.$ $0,1,2, \ldots, n\}$ is called a composition series for $F$ if $F_{i}$ is covered by $F_{i+1}$, for any $0 \leq i \leq n-1$, in ordered set $(F(A), \subseteq)$, i.e., $\left\{F_{i}: i=0,1,2, \ldots, n\right\}$ is a maximal $F$-chain. We denote the smallest length of a composition series for $F$ by $L(F)$ and if $F$ has no composition series, $L(F)=\infty$. It is clear that in any BL-algebra, $L(\{1\})=0$

Theorem 2.5 - [6]. Let A be an Artinian BL-algebra. Then Max $(A)$ is a finite set.

Definition 2.6 - [6]. A BL-algebra $A$ is called Noetherian (Artinian), if for every increasing (decreasing) chain of its filters $F_{1} \subseteq F_{2} \subseteq \ldots\left(F_{1} \supseteq F_{2} \supseteq \ldots\right)$, there exists $n \in \mathbb{N}$ such that $F_{i}=F_{n}$, for all $i \geq n$.

Theorem 2.7 - [6]. Let $A$ be a BL-algebra, $A$ is a Noetherian BL-algebra if and only if every filter of $A$ is finitely generated.

Lemma $2.8-$ [9]. Let $F$ and $G$ be two filters of $A$ such that $F \subseteq G$. Then the followings are equivalent:

(i) $F$ is covered by $G$.

(ii) $<F \cup\{x\}>=G$, for any $x \in G-F$.

(iii) $<x / F>=G / F$, for any $x \in G-F$.

Proposition 2.9 - [9]. Let $F$ and $G$ be two filters of BL-algebra $A$ such that $F \subset G$ and $G$ has a composition series, then $L(F)<L(G)$.

Theorem 2.10 - [9]. Let $F$ be a filter of $A$ such that $L(F)=n$, for some $n \in \mathbb{N}$. Then length of any composition series for $F$ is $n$. 
Definition 2.11 — [2]. The intersection of all maximal filters of a BL-Algebra $A$ is called radical of $A$ and denoted by $\operatorname{Rad}(A)$.

Definition $2.12-[6]$. Let $A_{1}, A_{2}, A_{3}$ be BL-algebras. A sequence $1 \longrightarrow A_{1} \stackrel{\varphi}{\longrightarrow} A_{2} \stackrel{\Psi}{\longrightarrow}$ $A_{3} \longrightarrow 1$ is called a short exact sequence of BL-algebras, if $\varphi$ is a one to one homomorphism, $\Psi$ is an onto homomorphism and $\operatorname{ker} \Psi=I_{m}(\varphi)$. Also, by [9], let $A_{1}, A_{2}, A_{3}$ be BL-algebras and $\varphi: A_{1} \longrightarrow A_{2}$ and $\psi: A_{2} \longrightarrow A_{3}$ be two homomorphism. By a weak exact sequence of BL-algebra we mean a sequence $A_{1} \stackrel{\varphi}{\longrightarrow} A_{2} \stackrel{\Psi}{\longrightarrow} A_{3}$ of BL-algebras such that $\operatorname{ker}(\psi) \subseteq \operatorname{Im}(\varphi)$.

Theorem 2.13 - [9]. Let $A_{1}, A_{2}, A_{3}$ be BL-algebras and $A_{1} \stackrel{\varphi}{\longrightarrow} A_{2} \stackrel{\Psi}{\longrightarrow} A_{3}$ be a weak exact sequence such that $\varphi$ is one to one and $\Psi$ is onto. Then $A_{2}$ is Noetherian (Artinian) BL-algebra if and only if $A_{1}$ and $A_{3}$ are Noetherian (Artinian).

Lemma 2.14 - [6]. Let $A$ be a Noetherian (Artinian) BL-algebra and $F \in F(A)$. Then $\frac{A}{F}$ is a Noetherian (Artinian) BL-algebra.

Theorem 2.15 - [6]. Let $A$ be a BL-algebra. Then $A$ is a Artinian BL-algebra if and only if every non-empty set of filters of $A$ has a minimal element.

Definition 2.16 - [4]. Let $A$ be a BL-Algebra. A proper deductive system of $A$ is called essential if $D \cap E \neq\{1\}$, for any deductive system $E \neq\{1\}$ of $A$. In particular, for an essential deductive system $D, D \neq\{1\}$.

From [7, 8], for any non-void subset $B$ of a BL-algebra $A$, a set ${ }^{\perp} B=\{x \in B \mid b \vee x=1$, for all $b \in A\}$ is called co-annihilator of $B$ and if $D$ is an essential deductive system of $A$, then ${ }^{\perp} D$ is a proper deductive system of $A$. Therefore, by Proposition 20 of [7], $D \cap^{\perp} D=\{1\}$, hence ${ }^{\perp} D=\{1\}$.

\section{NoEtherian AND Artinian ON BL-ALGEBRAS}

In this section, regarding to the definitions of Noetherian (Artinian), composition series, and short sequence BL-algebra and using above mentioned theorems, we drive theorems and obtain results of one to one, onto, and finitely generated BL-algebra, in a short exact sequence of BL-algebras and obtain the relation between Noetherian, Artinian, composition series, and short exact sequences of BL-algebras.

Corollary 3.1 - From [6], we conclude that, if $A$ be a BL-algebra, then the following statements are equivalent:

(i) $A$ is a Noetherian BL-algebra.

(ii) every collection of filters of $A$ has a maximal element. 
(iii) every filter of $A$ is finitely generated.

ProOF : (i) $\Longrightarrow$ (ii) Let $\left\{F_{i}\right\}_{i \in \mathbb{N}}$ be a collection of filters of $A$. If $F_{i_{1}}$ has no maximal element, hence there exists $F_{i_{2}}$ such that $F_{i_{1}} \subset F_{i_{2}}$ and if $F_{i_{2}}$ has no maximal element, so there exists $F_{i_{3}}$ such that, $F_{i_{2}} \subset F_{i_{3}}$. If we continue this procedure, we get $F_{i_{1}} \subset F_{i_{2}} \subset F_{i_{3}} \subset \ldots$, which is a contradiction, so $\left\{F_{i}\right\}_{i \in \mathbb{N}}$ has a maximal element.

(ii) $\Longrightarrow$ (i) Let every collection of filters of $A$ has a maximal element, and let $F_{1} \subseteq F_{2} \subseteq \ldots$ be an increasing chain of filters of $A$. Let $B=\left\{F_{i}: i \in \mathbb{N}\right\}$ be a nonempty set of filters of $A$. Since $B$ has a maximal element like $F_{n}$, so for all $i \geq n, F_{i}=F_{n}$. Hence $A$ is a Noetherian BL-algebra (similarly for Artinian BL-algebra).

(i) $\Longrightarrow$ (iii) Let $A$ be a Noetherian BL-algebra and $F$ be a filter of $A$ which is not finitely generated and $a_{1} \in F$. If $<a_{1}>\notin F$, then there exists $a_{2} \in F$ such that $a_{2} \notin<a_{1}>$, hence $<a_{1}>\subset<$ $a_{1}, a_{2}>$. If one continues this procedure, can get $<a_{1}>\subset<a_{1}, a_{2}>\subset<a_{1}, a_{2}, a_{3}>\subset \ldots$, then the above chain is nonstop and this is a contradiction. Therefore, $F$ is finitely generated filter of $A$.

(iii) $\Longrightarrow$ (i) Let $F_{1} \subseteq F_{2} \subseteq \ldots \subseteq F_{n} \subseteq \ldots$ be an increasing chain of filters of $A$, so $B=\cup_{i=1}^{\infty} F_{i}$ is a filter of $A$. Since $B$ is finitely generated, we have $\cup_{i=1}^{k} F_{i}=<a_{1}, a_{2}, \ldots, a_{k}>$, whereas, for all $i \neq j, a_{i} \in F_{k}, a_{j} \in F_{k}, a_{j} \in F_{m}$. Thus, there exist $F_{n}$ such that $\left\langle a_{1}, a_{2}, \ldots, a_{k}\right\rangle \subseteq F_{n}=F_{n+1}$. Therefore, there exists $m \in \mathbb{N}$ and there exist $a_{1}, a_{2}, \ldots, a_{k} \in F_{m}$, such that, $F_{m}=F_{m+1}=\ldots$, hence $A$ is a Noetherian BL-algebra.

Theorem 3.2 - Let $A$ be a BL-algebra and $F$ be a filter of $A$ which has a composition series and $L(F)=n$, then every strict chain of $F$ has $\leq n$ terms.

Proof : Let $\{1\}=\dot{G}_{0} \subset \dot{G}_{1} \subset \cdots \subset \dot{G}_{k-1} \subset \dot{G}_{k}=G$, be an strict chain of filters of $F$, then $0=L\{1\}=L\left(G_{0}\right) \subset L\left(\dot{G}_{1}\right) \subset \cdots \subset L\left(G_{k-1}^{\prime}\right) \subset L\left(\dot{G}_{k}\right)=L(G)$, hence $L(G) \geq k$ and if $\mathrm{G} \subset F$. Define $n \leq k$, by Proposition 2.9, we have $L(G)<L(F)$, then $k \leq n$, hence $k=n$.

Theorem 3.3 - Let $A$ be a BL-algebra and $F$ be a filter of $A$ which has a composition series and $L(F)=n$. Then every strict chain of length $n$ is a composition series.

PROOF : Let $\{1\}=F_{0} \subset F_{1} \subset \cdots \subset F_{i-1} \subset F_{i} \subset \cdots \subset F_{n}=F$ be an $F$-chain (strict chain) which is not composition series. This means that, there exists $i \in \mathbb{N}$ such that $F_{i-1}$ is not covered by $F_{i}$, So there exists $K_{i} \neq\{1\}$ such that $F_{i-1} \subset K_{i} \subset F_{i}$. Then we have $\{1\}=F_{0} \subset F_{1} \subset \cdots \subset$ $F_{i-1} \subset K_{i} \subset F_{i} \subset \cdots \subset F_{n}=F$, with length equal to $(n+1)$. This contradicts Theorem 2.7 , so this $F$-chain is a compositions series.

Proposition 3.4 - Let $A$ be a BL-algebra and $F$ be a filter of $A$ which has a composition series 
of length $n$. Then every strict chain of filter $F$ can be extended to a composition series.

Proof : Let $\{1\}=F_{0} \subset F_{1} \subset F_{2} \subset \ldots F_{m}=F$ be an strict chain of filter $F$. If $m=n$, then this strict chain is composition series. If $m<n$ then this chain is not composition series because by Theorem 2.10, any two composition series have the same length. So there exists $i \in \mathbb{N}$ such that, $H_{i} \neq\{1\}$ which, $F_{i-1} \subset H_{i} \subset F_{i}$, hence we have $\{1\}=F_{0} \subset F_{1} \subset \cdots \subset F_{i-1} \subset H_{i} \subset F_{i}=F$, this chain has length $(m+1)$. By continuing this process, we get a chain and finally by adding $(n-m)$ new terms, we get $(n-m)+m=n$. Hence this chain has length $n$, so by Theorem 3.3, is a composition series.

Theorem 3.5 - Let A be a BL-algebra. If A is Noetherian BL-algebra, then A has composition series.

Proof : Let $A$ be a BL-algebra and $A$ be Noetherion BL-algebra. Let $F_{1}$ be a minimal filter of $A$, then $\frac{F_{2}}{F_{1}}$ is a minimal filter of $\frac{A}{F_{1}}$. So $\{1\}=F_{0} \subset F_{1} \subset F_{2}$. Let $\frac{F}{F_{n-1}}$ be a minimal filter of $\frac{A}{F_{n-1}}$, hence $\{1\}=F_{0} \subset F_{1} \subset F_{2} \subset \cdots \subset F_{n-1} \subset F_{n} \subset \ldots$ Since $A$ is Noetherian there exists $n \in \mathbb{N}$ such that $F_{i}=F_{n}$, for all $i \geq n$, so $A$ has a composition series.

Lemma 3.6 - Let $A$ be a Artinian BL-algebra. Then $\operatorname{Rad}(A)=\bigcap_{i=1}^{k} F_{i}$ where $F_{i}$ are maximal filters of $A$ and $k \in \mathbb{N}$.

PROOF : Let $F_{1} \cap F_{2} \cap \cdots \cap F_{k}$ be an intersection of finitely many minimal of maximal filters of $A$, then for every maximal filters $M$ of $A$, we have $F_{1} \cap F_{2} \cap \cdots \cap F_{k} \cap M=F_{1} \cap F_{2} \cap \cdots \cap F_{k}$ so $\bigcap_{i=1}^{k} F_{i}$ $\subseteq M$, hence

$$
\operatorname{Rad}(A)=\bigcap_{i=1}^{k} F_{i}
$$

Lemma 3.7 - Let $A_{1}, A_{2}$ be two subalgebras of BL-algebra of $A$ and $A_{1} \subseteq A_{2}$. Then $A_{2}$ is Noetherian (Artinian) if and only if $A_{1}$ and $\frac{A_{2}}{A_{1}}$ are Noetherian (Artinian).

ProOF : The sequence $1 \longrightarrow A_{1} \stackrel{i}{\longrightarrow} A_{2} \stackrel{\pi}{\longrightarrow} \frac{A_{2}}{A_{1}} \longrightarrow 1$ is a short exact sequence, because $i$ is one to one homomorphism, then $\pi$ is an onto homomorphism and $I_{m} i=k e r \pi$. Now, if $A_{1}$ and $\frac{A_{2}}{A_{1}}$ are Noetherian (Artinian), then by Theorem 2.13, $A_{2}$ is Noetherian (Artininan). Conversely, let $A_{2}$ be a Noetherian (Artinian). Since the sequence $1 \longrightarrow A_{1} \stackrel{i}{\longrightarrow} A_{2} \stackrel{\pi}{\longrightarrow}$
$\frac{A_{2}}{A_{1}} \longrightarrow 1$ is a short exact sequence and $I_{m} i=k e r \pi$ then by Theorem $2.13, A_{1}$ and $\frac{A_{2}}{A_{1}}$ are Noethe-
rian (Artinian).

Lemma 3.8 : Let $A$ be a Noetherian(Artinian) BL-algebra and $B$ be a BL-algebra and $f: A \longrightarrow B$ 
be a BL-homomorphism. Then $f(A)$ is a Noetherian(Artinian) BL-algebra.

PROOF : Let $f\left(F_{1}\right) \subseteq f\left(F_{2}\right) \subseteq \cdots \subseteq f\left(F_{n}\right) \subseteq \ldots$ be an increasing chain of filters of $f(A)$, where $F_{i}$ are filters of $A$. Since $A$ is Noetherian BL-algebra and $F_{1} \subseteq F_{2} \subseteq \cdots \subseteq F_{n} \subseteq \ldots$ is an increasing chain of filters of $A$, so there exists $n \in \mathbb{N}$ such that $F_{i}=F_{n}$, for all $i \geq n$. Then $f\left(F_{i}\right)=f\left(F_{n}\right)$, for all $i \geq n$, so $f(A)$ is a Noetherian BL-algebra (similarly for Artinian BL-algebra).

Theorem 3.9 - Let $A$ be an Artinian BL-algebra and $f: A \longrightarrow A$ be a one to one BLhomomorphism. Then $f$ is an onto BL-homomorphism.

Proof : Suppose $f$ is not an onto BL-homomorphism, i.e., $A \supset f(A)$. Since $f$ is one to one, so $f(A) \supset f^{2}(A)$. We also have $f^{n-1}(A) \supset f^{n}(\mathrm{~A})$ for all $n \geq 2$. This means that $A \supset f(A) \supset$ $f^{2}(A) \supset \ldots \supset f^{n}(A) \supset \ldots$ is a decreasing chain of filters of $A$. This chain is not stationary, because, if there exists $k \in \mathbb{N}$ such that $f^{k+1}(A)=f^{k}(A)$, then by the injectivity of $f$, there exists a map $g: A \longrightarrow A, g(f(A))=I_{A}$, thus $g\left(f^{k+1}(A)\right)=g\left(f^{k}(A)\right)$, i.e., $f^{k}(A)=f^{k-1}(A)$. By continuing this procedure, we get $f(A)=A$, so which is a contradiction. Therefore, the above chain is not stationary and hence $A$ is not Artinian BL-algebra. It is a contradiction with hypothesis, hence $A=f(A)$ and $f$ is an onto BL-homomorphism.

Theorem 3.10 - Let A and B be two BL-algebras and $h: A \longrightarrow B$ is an onto BL-homomorphism. If $A$ is an Artinian BL-algebra, then $B$ is an Artinian BL-algebra.

Proof : First we know that for any filter $F$ of $B, h^{-1}(F)$ is a filter of $A$, because, let $x, y \in$ $h^{-1}(F)$, then $h(x), h(y) \in F$. Since $F$ is a filter of $B$, so $h(x) \odot h(y) \in F$, i.e., $h(x \odot y) \in F$ and hence, $x \odot y \in h^{-1}(F)$. Let $x \in h^{-1}(F), y \in A$ and $x \leq y$, then $h(x) \in F$. By [8, Remark 10] if $x, y \in A, x \leq y$, then $h(x) \leq h(y)$. Since $h(x) \in F, h(y) \in B$ and $F$ is a filter of $B$, so $h(y) \in F$, i.e., $x \in h^{-1}(F)$. Let $F_{1} \supseteq F_{2} \supseteq \ldots$ be a decreasing chain of filters of $B$, then $h^{-1}\left(F_{i}\right)$, $i \geq 1$ are filters of $A(*)$. Since $h$ is onto, $h^{-1}\left(F_{1}\right) \supseteq h^{-1}\left(F_{2}\right) \supseteq \ldots$ is a decreasing chain of filters of $A$. By the hypothesis, $A$ is Artinian BL-algebra, then there exists $i \in \mathbb{N}, h^{-1}\left(F_{i}\right)=h^{-1}\left(F_{n}\right)$ for all $n \geq i$. The fact that $h$ is an onto BL-homomorphism, we get $h\left(h^{-1}\left(F_{i}\right)\right)=h\left(h^{-1}\left(F_{n}\right)\right)$, for all $n \geq i$. Hence for all $n \geq i, F_{i}=F_{n}, B$ is Artinian BL-algebra.

Proposition 3.11 - Let $A$ and $B$ be two BL-algebras and $h: A \longrightarrow B$ be an onto BLhomomorphism. If $A$ is a Noetherian BL-algebra, then $B$ is Noetherian BL-algebra.

PROOF : Let $F_{1} \subseteq F_{2} \subseteq \ldots$ be an increasing chain of filters of $B$, then by $(*)$ in the proof of Theorem 3.10, for any $i \geq 1, h^{-1}\left(F_{i}\right)$, is a filter of $A$, therefore, by the surjectivity of $h, h^{-1}\left(F_{1}\right) \subseteq$ 
$h^{-1}\left(F_{2}\right) \subseteq \ldots$ is an increasing chain of filters of $A$. Since $A$ is a Noetherian BL-algebra, then there exists $i \in \mathbb{N}$, such that for all $n \geq i, h^{-1}\left(F_{i}\right)=h^{-1}\left(F_{n}\right)$. $h$ is an onto BL-homomorphism, so we get $h\left(h^{-1}\left(F_{i}\right)\right)=h\left(h^{-1}\left(F_{n}\right)\right)$, for all $n \geq i$. Hence for all $n \geq i, F_{i}=F_{n}$ and $B$ is a Noetherian BL-algebra.

Lemma 3.12 - Let $A_{1}, A_{2}$ and $A_{3}$ be BL-algebras and $1 \longrightarrow A_{1} \stackrel{\varphi}{\longrightarrow} A_{2} \stackrel{\Psi}{\longrightarrow} A_{3} \longrightarrow 1$ is a short exact sequence of BL-algebra, if every filters of $A_{1}$ and $A_{3}$ are finitely generated, then every filter of $A_{2}$ is finitely generated.

ProOF : Let $1 \longrightarrow A_{1} \stackrel{\varphi}{\longrightarrow} A_{2} \stackrel{\Psi}{\longrightarrow} A_{3} \longrightarrow 1$ be a short exact sequence of BL-algebra. By Theorem 2.7, since every filters of $A_{1}$ and $A_{3}$ is finitely generated, so $A_{1}$ and $A_{3}$ are Noetherian. Further by Theorem 2.13, since $A_{1}$ and $A_{3}$ are Noetherian, then $A_{2}$ is Noetherian BL-algebra. Hence by Theorem 2.7, every filters of $A_{2}$ is finitely generated.

Definition 3.13 - Let $A$ be a BL-algebra and $F_{i} \in \digamma(A) . A$ is called finitely embedded, if any intersection of filters reduces to, finite intersection, i.e., $\bigcap_{i \in I} F_{i}=F_{i_{1}} \bigcap \ldots \bigcap F_{i_{n}}$, such that $i_{1}, i_{2}, \ldots, i_{n} \in I$.

Proposition 3.14 - Let $A$ be a BL-algebra. $A$ is an Artinian BL-algebra if and only if for any $F \in \digamma(A) ; \frac{A}{F}$ is a finitely embedded BL-algebra.

Proof : Let $A$ be an Artinian BL-algebra. By Theorem 2.14, every quotation filter of $A$ is Artinian too, hence it is sufficient to show that $A$ is finitely embedded. Let $I, J$ be index sets and consider the element $\bigcap_{i \in I} F_{i}$, where each $F_{i}$ is filter of $A$. Let $\bigcap_{i \in J} F_{i}$ be a minimal (by Theorem 2.15 , one can show that every collection of filters of $A$ has a minimal element) among $\bigcap_{i \in K} F_{i}$, where the cardinal of $K$ is finite. Put $F=\left\{F_{i_{1}} \cap \ldots \bigcap F_{i_{n}}: i_{n} \in I\right\}$. Now we claim that $\bigcap_{i \in I} F_{i}=\bigcap_{i \in J} F_{i}$.

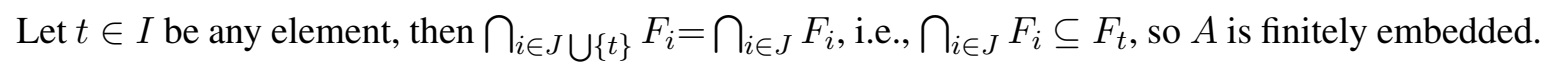

Conversely, let $F_{1} \supseteq F_{2} \supseteq \ldots \supseteq F_{n} \supseteq \ldots$., be a chain of $A$. Then consider the collection $\left\{\frac{F_{i}}{\bigcap_{i \in I} F_{i}}\right\}$ in $\frac{A}{\bigcap_{i \in I} F_{i}}$. Hence $\bigcap_{i \in I} \frac{F_{i}}{\bigcap_{i \in I} F_{i}}=\frac{F_{i_{1}}}{\bigcap_{i \in I} F_{1}} \bigcap \frac{F_{i_{2}}}{\bigcap_{i \in I} F_{2}} \cap \ldots \bigcap \frac{F_{i_{n}}}{\bigcap_{i \in I} F_{n}}=\frac{F_{i_{1}} \bigcap \ldots \bigcap F_{i_{n}}}{\bigcap_{i \in I} F_{i}}$ $=\frac{0}{\bigcap_{i \in I} F_{i}}=0$, so $F_{i_{1}} \bigcap \ldots \bigcap F_{i_{n}}=\bigcap_{i \in I} F_{i}$, let $i_{1} \subseteq i_{2} \subseteq \ldots \subseteq i_{n}$, i.e., $F_{i_{n}}=\bigcap_{i \in I} F_{i}$. Since $F_{i_{n}} \subseteq F_{i}$, then $F_{i_{n}}=F_{i_{n+1}}=\ldots$, thus $A$ is Artinian BL-algebra.

By Theorem 2.15, Corollary 3.1, and Proposition 3.14, the following conclusion holds:

Corollary 3.15 — Let $A$ be a BL-algebra. Then the following conditions are equivalent:

(i) $A$ is an Artinian (Noetherian) BL-algebra.

(ii) Every collection of filters of $A$ has a minimal (maximal) element. 
(iii) $A$ is finitely embedded (every filter of $A$ is finitely generated).

Proof : (i) $\Longrightarrow$ (ii) Let $A$ be an Artinian BL-algebra. So, by Theorem 2.15, every Collection of filters of $A$ has a minimal element.

(ii) $\Longrightarrow$ (i) Let every Collection of filters of $A$ has a minimal element. Then, by Theorem 2.15, $A$ is an Artinian BL-algebra.

(iii) $\Longrightarrow$ (i) Let $A$ be finitely embedded. It is clear that for any $F \in \digamma(A), \frac{A}{F}$ is finitely embedded then, by Proposition 3.14, $A$ is an Artinian BL-algebra.

(i) $\Longrightarrow$ (iii) Let $A$ be an Artinian BL-algebra. Then by Proposition 3.14, for any $F \in \digamma(A), \frac{A}{F}$ is finitely embedded. Therefore, by the proof of Proposition 3.14, $A$ is a finitely embedded BL-algebra. Similarly, Corollary 3.15, holds for Noetherian BL-algebra according to the Corollary 3.1.

Proposition 3.16 - Let $A$ and $B$ be two BL-algebras and $f: A \longrightarrow B$ be a BL-homomorphism. If $D$ is an essential deductive system of $B$, then $f^{-1}(D)$ is an essential deductive system of $A$.

Proof : Let $D$ be an essential deductive system of $B$ and $M \neq\{1\}$ be any deductive system of $A$. If $f(M)=\{1\}$, then $M \subseteq f^{-1}(D)$ and $M \cap f^{-1}(D) \neq\{1\}$. Otherwise, if $f(M) \neq\{1\}$ then $f(M) \cap D \neq\{1\}$. Since $D$ is an essential deductive system of $B$, we have $f^{-1}(f(M) \cap D)=$ $M \cap f^{-1}(D) \neq\{1\}$. Hence $f^{-1}(D)$ is an essential deductive system of $A$.

Proposition 3.17 - Let $A$ and $B$ be two BL-algebras such that $A \subseteq B$ and $D_{1}, D_{2}$ be essential deductive systems of $A$ and $B$ respectively. Then $D_{1} \cap D_{2}$ is an essential deductive system of $A \cap B$.

Proof : Let $D \neq\{1\}$ be an essential deductive system of $A \cap B$. Since $D_{2}$ is an essential deductive system of $B$, then $D \cap D_{2} \neq\{1\}$. We obtain $\{1\} \neq D \cap D_{2} \subseteq D$, because, $D_{2}$ is an essential deductive system of $B$ and $\{1\} \neq D \subseteq A \cap B \subseteq B$. Since $D_{1}$ is an essential deductive system of $A$, then $D_{1} \cap\left(D_{2} \cap D\right) \neq\{1\}$. Thus $\left(D_{1} \cap D_{2}\right) \cap D \neq\{1\}$, hence $D_{1} \cap D_{2}$ is an essential deductive system of $A \cap B$.

Proposition 3.18 - Let $A_{1}, A_{2}$ and $A_{3}$ be BL-algebras such that $A_{1} \subseteq A_{2} \subseteq A_{3}$. Then $D$ is an essential deductive system of $A_{3}$ if and only if $D$ is an essential deductive system of $A_{1}$ and $A_{2}$.

ProOF : Let $D \neq\{1\}$ be an essential deductive system of $A_{3}$, then for any deductive system $E \neq\{1\}$ of $A_{3}, D \cap E \neq\{1\}$ and for any deductive system $G \neq\{1\}, G \subseteq A_{2} \subseteq A_{3}$, we have $D \subseteq G \neq\{1\}$. So $D \neq\{1\}$ is an essential deductive system of $A_{2}$. Similarly, for any deductive system $K \neq\{1\}$ of $A_{1}$, since $A_{1} \subseteq A_{2}$ and $D \cap K \neq\{1\}$, then $D$ is an essential deductive system of $A_{1}$. 
Conversely, let $D \neq\{1\}$ be an essential deductive system of $A_{1}$ and $A_{2}$, then for any deductive system $E \neq\{1\}$ of $A_{2}, D \cap E \neq\{1\}, D \cap E \subseteq D$. Since $D$ is an essential deductive system of $A_{1}$, then for any deductive system $G \neq\{1\}$ of $A_{1}, G \cap(D \cap E)=(G \cap D) \cap E \neq\{1\}$ hence, $G \cap D \neq\{1\}$. Since $A_{1} \subseteq A_{2} \subseteq A_{3}$, and for any deductive systems $E \neq\{1\}, D \neq\{1\}$, we obtain the deductive systems $G \cap E \neq\{1\}$ and $(G \cap E) \cap D \neq\{1\}$. Therefore, $D$ is an essential deductive system of $A_{3}$.

\section{ACKNOWLEDGEMENT}

The authors are extremely grateful to the editor and the referee for his/her valuable comments and helpful suggestions which help to improve the paper.

\section{REFERENCES}

1. R. A. Borzooei and A. Paad, Generalization of integral filters in BL-algebras and $n$-fold integral BLalgebras, Afrika Mat., 26(7-8) (2014), 1299-1311.

2. F. Esteva and L. Godo, Monoidal $t$-norm based logic: Towards a logic for left-continuous $t$-norms, $F u z z y$ Sets Syst., 124 (2001), 271-288.

3. P. Hájek, Metamathematics of fuzzy logic, Kluwer Academic Publishers, Dordrecht, (1998), ISBN: 07923-5238-6.

4. C. S. Hoo, Semilocal MV-algebras, Math Japonica., 40 (1994), 451-453.

5. L. Leustean, Representations of many-valued algebras, Ph.D Thesis, Faculty of Mathematics and Basic Science, University of Bucharest, Bucharest, Romania (2003).

6. S. Motamed and J. Moghaderi, Noetherian and Artininan BL-algebras, Soft Comput., 16 (2012), 19891994.

7. E. Turunen, BL-algebras of basic fuzzy logic, Mathware Soft Comput., 6 (1999), 49-61.

8. E. Turunen, Mathematics behind fuzzy logic, Physica-Verlag, Heidelberg, (1999), ISBN: 3-7908-1221-8.

9. O. Zahiri, Chain conditions on BL-algebras, Soft Comput., (2013), doi 10.1007/soo 500-013-1099-2. 